
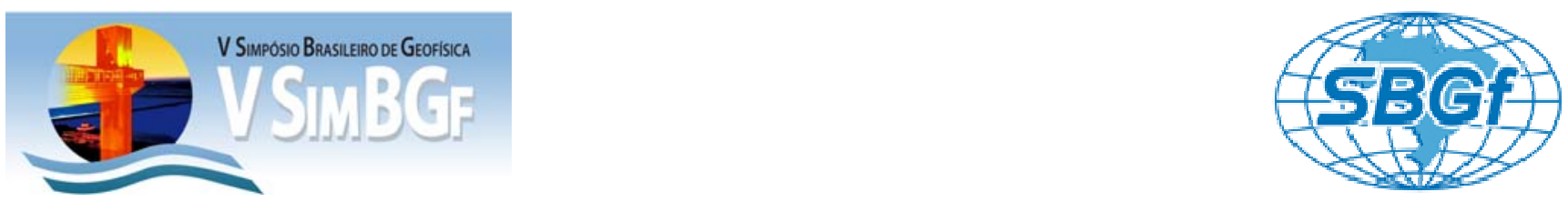

\title{
Aplicabilidade de Equipamento de Aquisição Sísmica Wireless em Território Brasileiro
}

Ricardo de Faria Barcelos ${ }^{1}$, ricardo.barcelos@georadar.com.br

Douglas Iceri Lasmar ${ }^{1}$, Jarbas Ribeiro de Oliveira ${ }^{1}$, Bruno Henrique de Moura Martins ${ }^{1}$

${ }^{1}$ Georadar Levantamentos Geofísicos S.A.

Copyright 2012, SBGf - Sociedade Brasileira de Geofísica

Este texto foi preparado para a apresentação no V Simpósio Brasileiro de Geofísica Salvador, 27 a 29 de novembro de 2012. Seu conteúdo foi revisado pelo Comitê Técnico do V SimBGf, mas não necessariamente representa a opinião da SBGf ou de seus associados. É proibida a reprodução total ou parcial deste material para propósitos comerciais sem prévia autorização da SBGf.

\section{Resume}

With the technology improvements in hi-density power sources, electronic reliability, survey design and processing, the use of cable free systems has increased in the last years. In Brazil one of the new generation wireless system is been used since 2007 and this paper will provide a description of its use, advantages and disadvantages in seismic surveys. The results from acquired surveys, applicability tests and quality control procedures are presented and, as a last topic, alternatives of optimizations, like comparison from different battery's technologies and operational plans, to increase production and at the same time decrease costs are evaluate.

\section{Introdução}

Trabalhos de aquisição sísmica com dispositivos wireless vêm ganhando espaço à medida que dificuldades técnicas são superadas pelo desenvolvimento de novos equipamentos e tecnologias. Mougenot (2010) conclui que sistemas híbridos, com e sem cabos, serão em curto prazo a opção para vencer barreiras ambientais. Lansley (2012) evidencia diferentes situações onde diferentes sistemas de aquisição (com cabo, sem cabo e híbrido) apresentam melhores resultados operacionais.

Segundo Caldwell (2010) vários trabalhos sobre o desenvolvimento de sistemas wireless foram publicados nos últimos anos, antecipando suas vantagens (maior produtividade; mobilidade, espaçamento flexível entre receptores, redução no número de trabalhadores expostos a riscos e conseqüentemente melhor desempenho de QSMS), bem como suas desvantagens (tempo de vida e gerenciamento de baterias, aquisição em non-real-time, facilidade de perda ou roubo de canais e necessidade de sincronização por GPS). Caldwell detalha ainda que apesar dos sistemas wireless possuírem melhor relação custo benefício, a mudança não será imediata, pois ainda é necessário transpor o desconforto causado pela impossibilidade de visualização dos resultados adquiridos em tempo real.

O presente trabalho tem o objetivo de apresentar técnicas de otimização operacional, bem como, resultados com o equipamento de aquisição sísmica wireless em território brasileiro, especialmente em regiões de densa vegetação onde o sinais GPS utilizados para sincronismo das unidades podem ser insuficientes.

Para avaliação do equipamento wireless utilizado (Unite/Sercel) foram avaliados operacionalmente projetos desenvolvidos pela GEORADAR LEVANTAMENTOS GEOFÍSICOS S.A., conforme representação na fig. 1, nas Bacias do Acre, Paraná, Potiguar, Sergipe/Alagoas, bem como testes e ensaios nas Bacias do Amazonas e São Francisco.

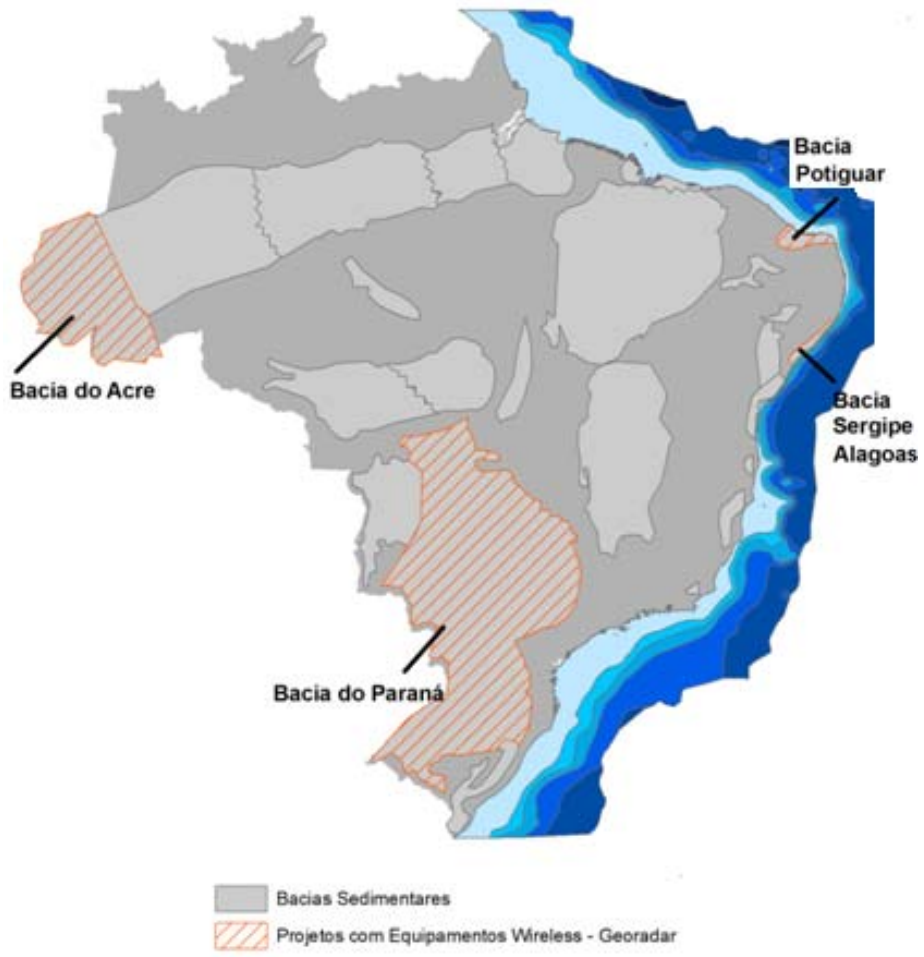

Figura 1 - Bacias com levantamentos sísmicos utilizando equipamentos wireless - Georadar Levantamentos Geofísicos S.A.

Para melhor detalhamento das áreas avaliadas, com intuito de pesquisas em bacias sedimentares, obras de infra estrutura e testes operacionais, a fig. 2 apresenta (marcados em vermelho) os estados brasileiros onde dados com sistema wireless foram coletados. 


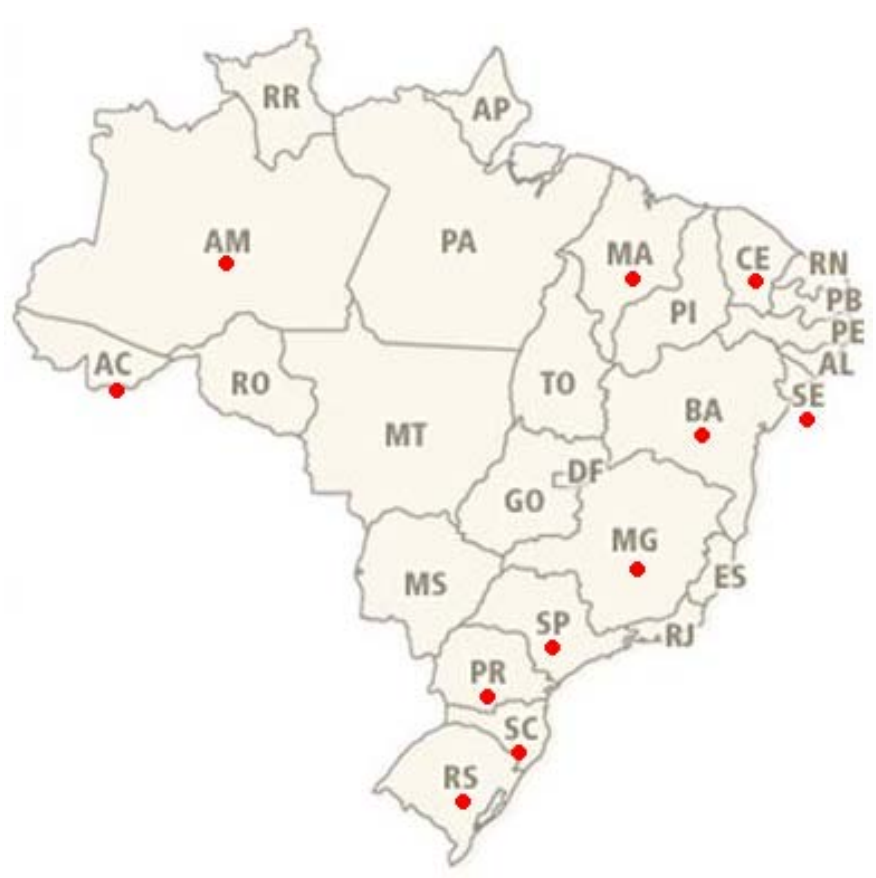

Figura 2 - Estados com estudos sísmicos utilizando equipamentos wireless - Georadar Levantamentos Geofísicos S.A.

\section{Descrição do Sistema e Procedimentos de Recuperação de Dados}

O sistema de aquisição sísmica wireless utilizado possui dois modos de atuação, autônomo e tempo real. Para o modo em tempo real são necessários equipamentos para transmissão de dados como estruturas e antenas adicionais aos canais receptores (apresentados na fig. 3) e sensores usualmente utilizados, já para o modo autônomo a configuração é a mesma dos sistemas com cabos (unidade digitalizadora e sensor).

No modo de aquisição em tempo real a utilização de estruturas e antenas (apresentadas na fig. 4) torna o sistema ineficiente operacionalmente, já que adicionalmente ao aumento de trabalhadores expostos a risco, as repetidoras são de curto alcance e, quando incorretamente posicionadas/locadas restringem outras formas de coletas de dados como "coletores portáteis" operados por uma única pessoa e/ou veículo (terrestre, fluvial ou aéreo) que se desloque próximo aos receptores.

No modo autônomo somente é necessário o espalhamento de canais receptores e sensores, no entanto, em momento posterior, os dados gravados devem ser coletados em intervalos regulares de tempo, tanto para que possam ser disponibilizados ao processamento quanto para continua avaliação das unidades autônomas em campo.

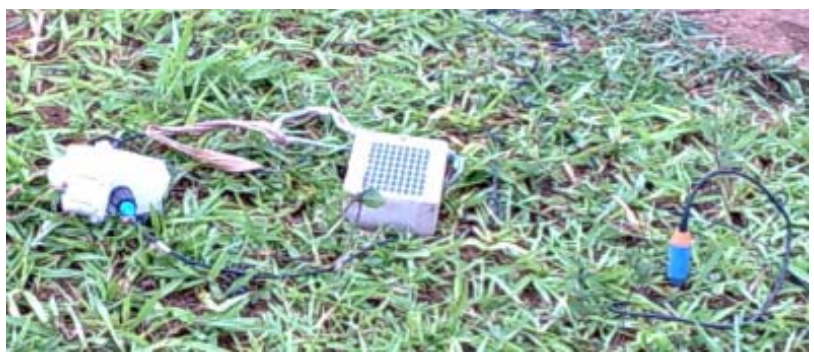

Figura 3 - Unidades de aquisição wireless, bateria externa e sensor (uma de seis bobinas).

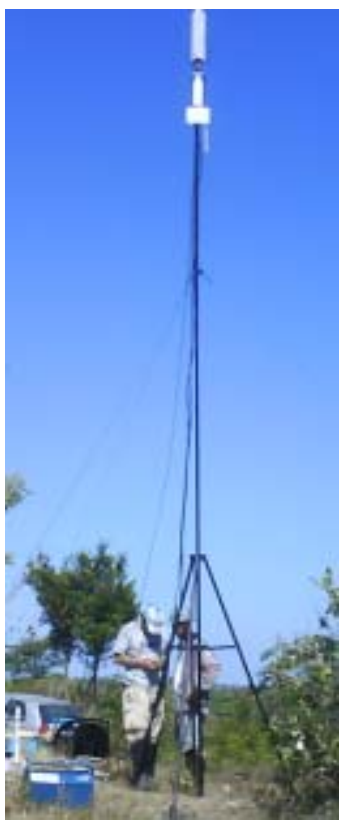

Figura 4 - Antena repetidora para utilização em tempo real

Os resultados mostrados a seguir foram obtidos com o equipamento em modo autônomo somado a medidas de controle de qualidade para minimizar o tempo de resposta e evitar possíveis ocorrências durante a aquisição.

Para garantir o controle de qualidade, todas as unidades receptoras são verificadas antes do início do registro. Adicionalmente todos os dados adquiridos são disponibilizados dentro do intervalo de 24 horas para verificação no controle de qualidade da equipe, possibilitando intervenções antes do recolhimento do material sismográfico.

\section{Operações em áreas de sombra}

Equipamentos sísmicos necessitam obter sincronismo entre as unidades receptores de gravação e fontes de energia (usualmente vibradores ou explosivos). Para solucionar este problema no equipamento wireless 
avaliado, cada unidade receptora possui um relógio GPS, como as unidades registram em tempo integral, cada amostra coletada é referenciada a um instante de tempo. O mesmo ocorre no sistema supervisório (ou sismógrafo), onde um relógio GPS referencia o momento em que cada fonte foi acionada. Em momento posterior, os dados de fonte e receptores são cruzados para coleta de dados. Sendo assim, para o início da gravação de dados é necessário que todas as unidades obtenham suficiente sinal de GPS, com o objetivo de sincronizar os relógios internos.

Com isso, a sua utilização em diversos ambientes proporciona questionamentos sobre a recepção de sinal, especialmente em locais remotos, com condições atmosféricas irregulares e densa vegetação.

Durante a aquisição de dados e testes realizados, a sincronização com o sinal de GPS foi possível mesmo em situações onde o sinal apresentou-se baixo (13\%). No Gráfico 1 é mostrada a variação do sinal de GPS. Observa-se que foi obtido um valor médio de 34\% para as unidades no lance do dispositivo.

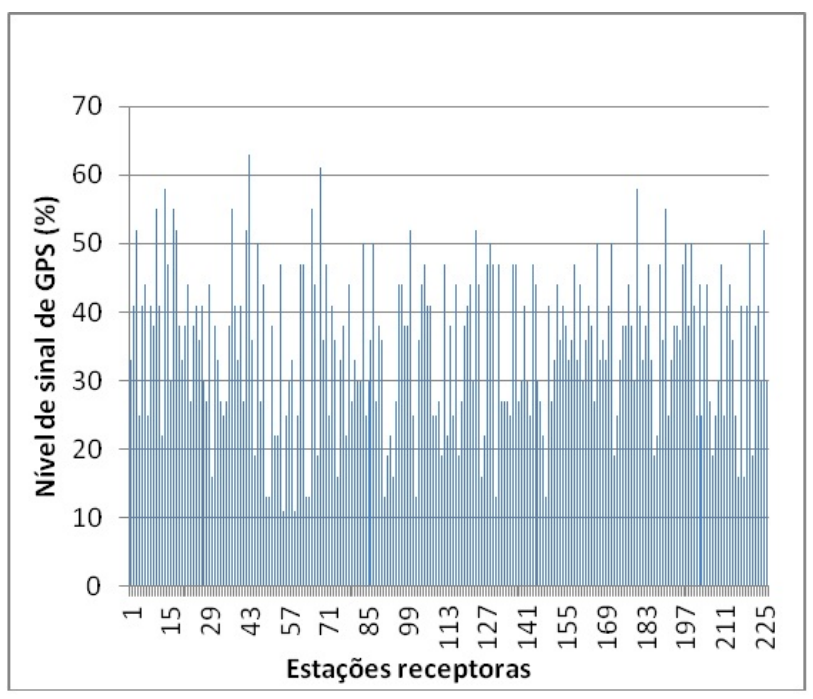

Gráfico 1 - Nível de sinal de GPS para estações. Fonte: Dados coletados nas unidades (RAU) na data 10/08/2012.

As unidades foram dispostas em pontos com diferentes densidades de vegetação, declividades e condições atmosféricas, todavia nenhum dos fatores foi suficiente para evitar o recebimento de sinal de GPS e respectivo sincronismo da unidade.

As fig. 3 e 4 demonstram exemplos de localização das unidades assim como sua vista superior e respectivo sinal de GPS.

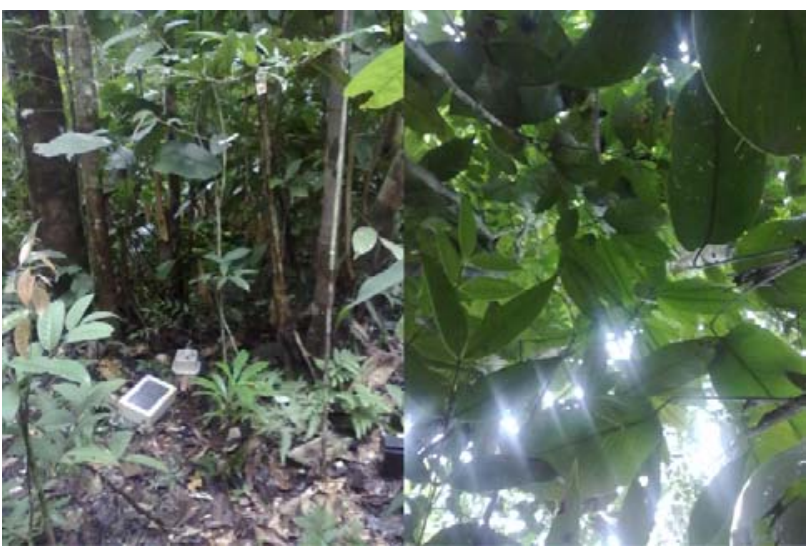

Figura 5 - Unidade 6053 - 47\% Sinal de GPS

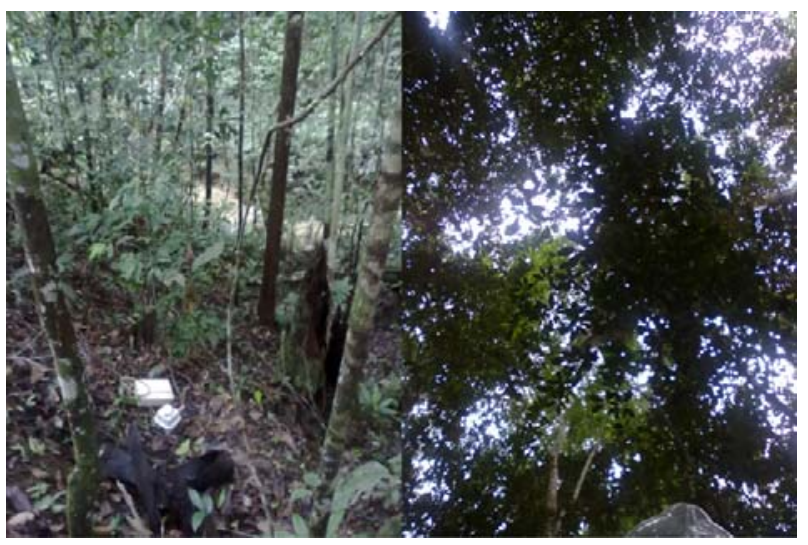

Figura 6 - Unidade 6480 - 33\% GPS

\section{Procedimentos para controle de qualidade}

Equipamentos wireless em modo autônomo não possibilitam visualização em tempo real de condições das unidades receptores, dados coletados, ou acompanhamento dos níveis de ruído em campo, com isso exige-se que procedimentos específicos sejam realizados para garantir o controle de qualidade nos registros. Destacam-se como pontos principais do controle de qualidade:

- Comunicação eficiente por rádio da equipes de registro, revisão, espalhamento e recolhimento;

- Revisão de canais antes e durante os registros;

- Coleta regular de dados, preferencialmente com intervalos menores que 24 horas;

- Posicionamento estratégico de revisores ao longo da linha de receptores;

Ressalta-se que o nível de confiabilidade dos dispositivos eletrônicos atuais aliados aos procedimentos citados 
acima possibilitaram obter aproveitamento de dados (traços) maior que 99\%.

\section{Otimização operacional}

Dois fatores importantes dominam a eficiência de operação de registro de dados, o primeiro deles é o peso por canal. Quanto maior o peso maior o número de trabalhadores necessários para o espalhamento e recolhimento dos equipamentos, assim como maiores os gastos com movimentações especialmente utilizando transporte aéreo.

O segundo fator determinante na eficiência é o tempo de autonomia dos unidades receptoras. Quanto menor o tempo de autonomia ou maior consumo de energia por canal maior será o número de substituições de baterias necessárias, o que pode inviabilizar a utilização de sistemas de aquisição wireless.

As baterias SLA (Sealed Lead-Acid) frequentemente são utilizadas para alimentação das unidades de aquisição em campo devido a seu baixo custo e disponibilidade no mercado local. Entretanto sua densidade de carga apresenta valores 10 vezes inferiores aos modelos compostos por íon Lítio. O que possibilitaria uma melhoria significativa em redução de peso e maior densidade energética.

Nos Gráficos 2 e 3 obtemos comparações entre sistemas de aquisição wireless e via cabo para levantamentos 2D e 3D respectivamente, assim como a utilização de dois modelos de baterias (SLA e íon Lítio).

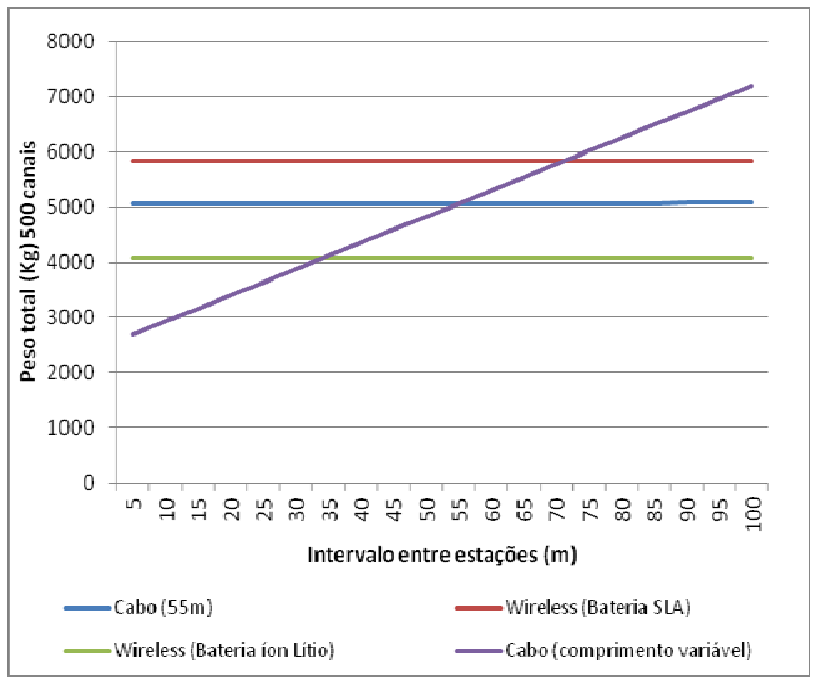

Gráfico 2 - Distribuição de peso para Aquisições 2D. Fonte: Dados retirados das especificações técnicas de equipamentos Sercel 428 e UNITE.

Verifica-se para aquisições em 2D que a partir de 35 metros entre estações a utilização de unidades wireless alimentadas por baterias íon Lítio apresentam menor peso por canal em relação ao sistema por cabos. Comportamento similar é obtido com espaçamento de $75 \mathrm{~m}$ entre estações, os quais sistemas wireless com baterias SLA obtêm melhor relação do que os sistemas via cabo.

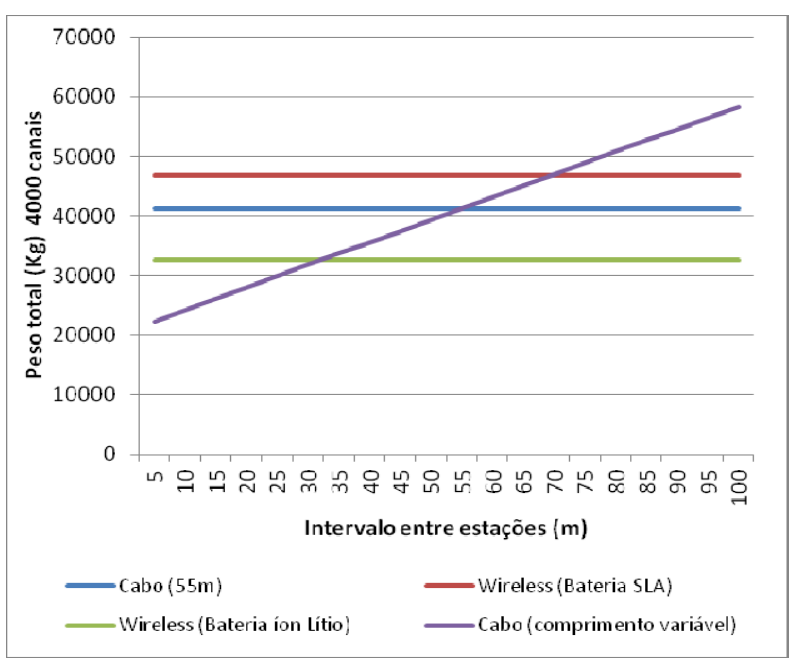

Gráfico 3 - Distribuição de peso para Aquisições 3D. Fonte: Dados retirados das especificações técnicas de equipamentos Sercel 428 e UNITE.

Para aquisições 3D resultados equivalentes foram obtidos, com pequenas variações devido ao aumento de equipamentos necessários para conexão entre linhas.

Reduções no peso e quantitativo de pessoal das equipes tomam maior importância em operações com transporte aéreo, como as realizadas em selva, onde o material das linhas de aquisição é deslocado paras clareiras mais próximas e, posteriormente, transportado para a linha seguinte a ser espalhada através de helicópteros, conforme representado na Fig. 7 .

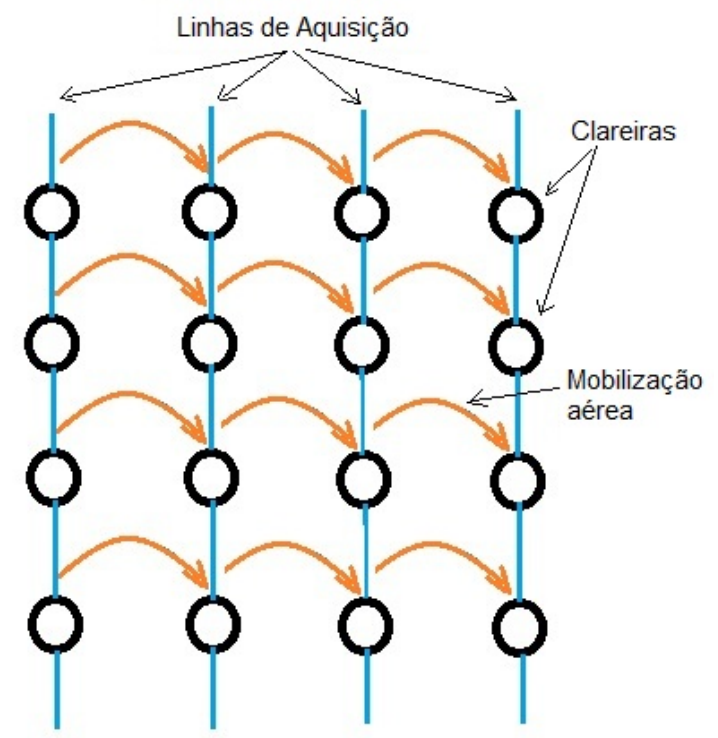


Figura 7 - Mobilização entre linhas de aquisição, operações em selva.

Os Gráficos 4, 5, 6 e 7 apresentam o número de mobilizações entre clareiras (pernadas de helicóptero) para diferentes espaçamentos entre estações receptoras (25 e 50m), capacidade energética das baterias (9 e 18 Ah) e diferente densidade energética (baterias SLA e Lítio).

Observa-se que para o espaçamento de receptores de $50 \mathrm{~m}$, e se a operação permitir a utilização de baterias de 9Ah (duração aproximada de 3.5 dias) existe uma redução significativa (cerca de 33\%) no número de vôos para transporte de material sismográfico.

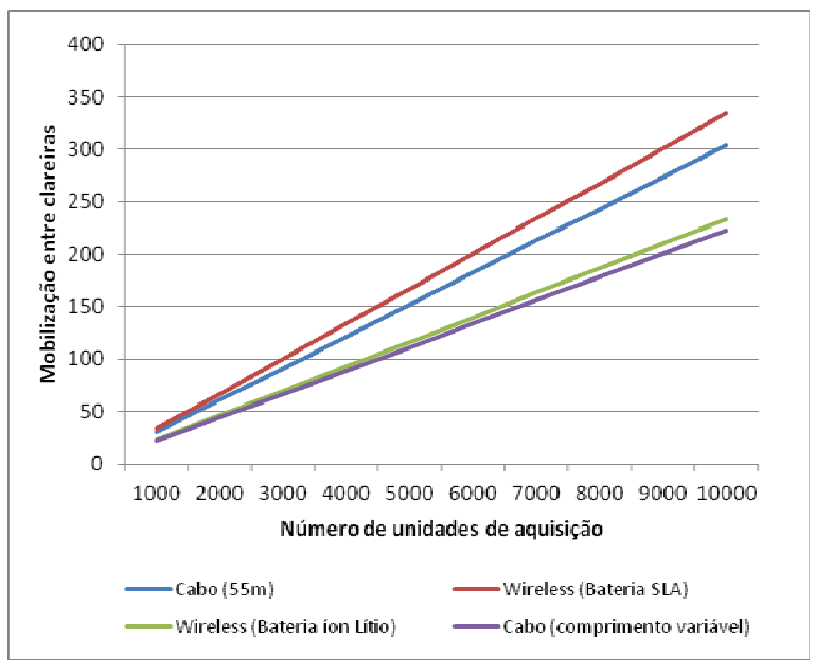

Gráfico 4 - Intervalo entre receptores de $25 m$ e bateria 9Ah.

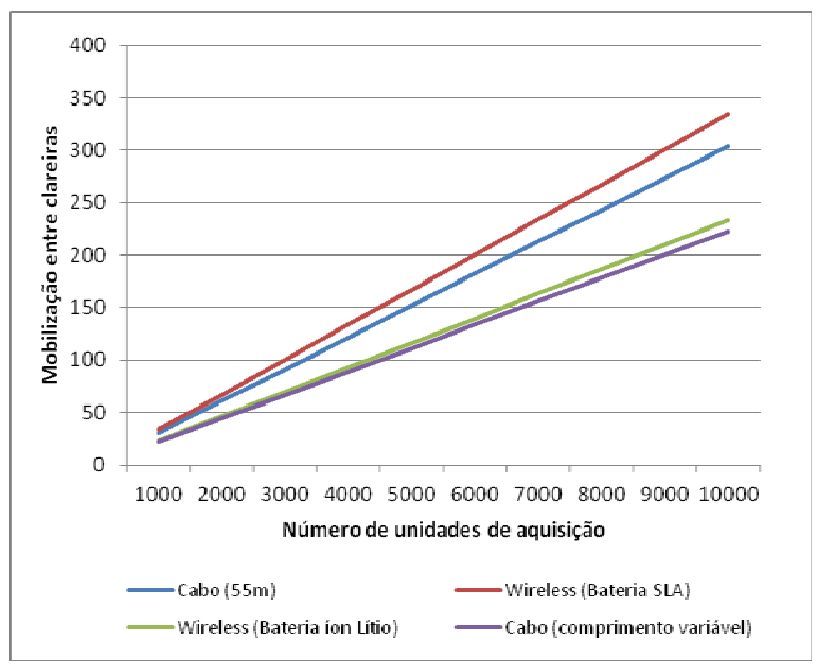

Gráfico 5 - Intervalo entre receptores de 25, bateria 18Ah.

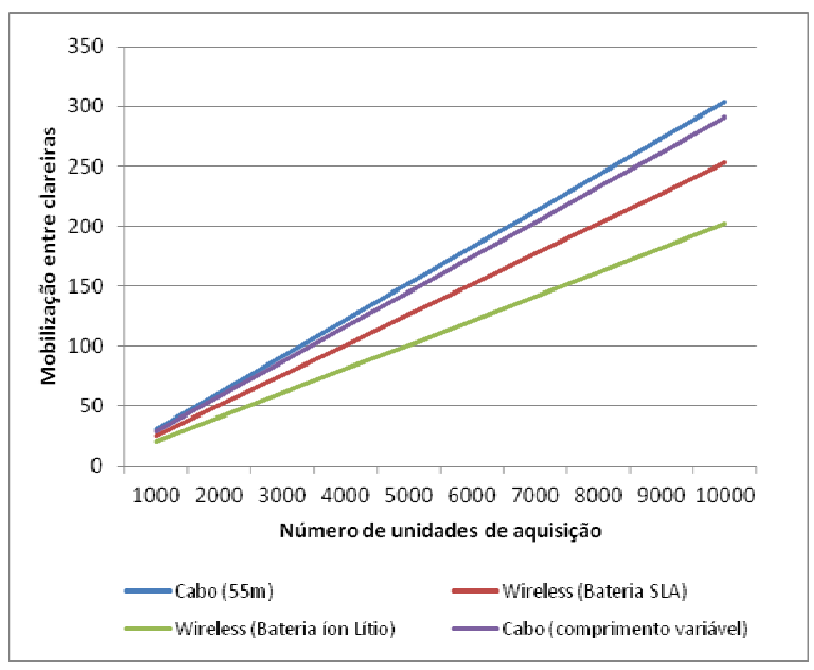

Gráfico 6 - Intervalo de receptores a 50m, bateria 9Ah

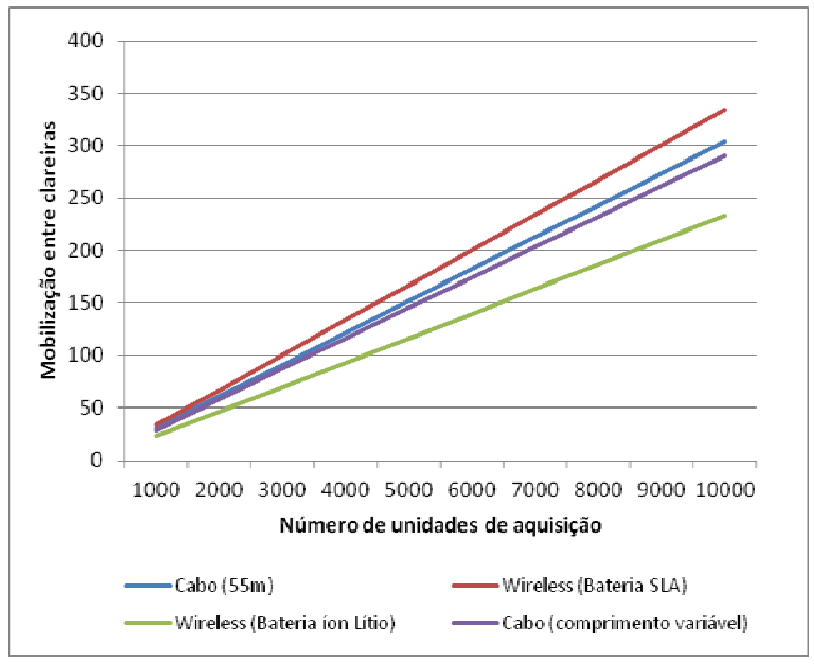

Gráfico 7 - Intervalo entre receptores de 50m, bateria $18 A h$

\section{Conclusões}

Sistemas de aquisição wireless apresentam vantagens operacionais com o distanciamento entre estações receptoras quando utilizados em conjunto com baterias de melhor densidade de carga.

Vantagens como redução significativa de peso por canal e consequentemente número total de integrantes da equipe, proporcionando melhores índices de QSMS e custos operacionais. Adicionalmente permitem a eliminação de paradas devido a problemas de transmissão de dados, comuns em sistemas via cabo.

Com a redução da distância entre estações receptoras, sistemas de aquisição via cabo apresentam melhores resultados com o benefício de monitoramento em tempo real de todas as unidades. 
Portanto, embora o sistema wireless Unite tenha apresentado resposta operacional positiva em 100\% das áreas testadas (11 estados brasileiros, incluído Acre e Amazonas com densa vegetação) para se optar entre aplicações de sistemas de aquisição wireless, via cabo ou híbridos devem ser realizadas avaliações das condições de área, critérios de segurança, objetivos e desenho do projeto.

\section{Referências}

Lansley, M., Laurin M. and Ronen S. [2008] Modern land recording systems: How do they weigh up? The leading Edge 27,888-984

Caldwell, Jack, Cable-less seismic offers real-world advantages: E\&P Magazine, April 2010, p. 46-48.

Mougenot, D. (2010) Land cableless systems: use and misuse. First Break 28(2),55-58.

Lansley, M. [2012] Cabled versus cable-less acquisition: making the best of both worlds in difficult operational environments. First Break p.97-102. 